quality of bowel preparation or caecal visualisation/intubated rates $(\mathrm{p}=0.93)$.

Conclusion Moviprep ${ }^{\circledR}$ was tolerated much better when compared to Klean-prep ${ }^{\mathbb{R}}$ in terms of side effects and the willingness to take the preparation again. Having a preparation that is well tolerated may help with patient compliance and improve colonoscopic examination.

Competing interests None Declared.

\section{PMO-202 USE OF THE BLATCHFORD SCORE TO IDENTIFY LOW-RISK UPPER GASTROINTESTINAL BLEEDS}

doi:10.1136/gutjnl-2012-302514b.202

B R Disney, ${ }^{2} \mathrm{R}$ Watson, ${ }^{2} \mathrm{~A}$ Blann, ${ }^{2} \mathrm{G}$ Lip, ${ }^{3} \mathrm{C}$ Tselepis, ${ }^{1} \mathrm{M}$ Anderson. ${ }^{1}$ Gastroenterology, Sandwell and West Birmingham Hospitals NHS Trust, Birmingham, UK; ${ }^{2}$ Cardiology, Sandwell and West Birmingham Hospitals NHS Trust, Birmingham, UK; ${ }^{3}$ Cancer Sciences, University of Birmingham, Birmingham, UK

Introduction Acute upper gastrointestinal bleeding is a medical emergency associated with a significant health burden and risk of mortality. A significant proportion of patients are admitted to hospital unnecessarily for endoscopy following presentation with acute upper gastrointestinal bleeding. The Blatchford score can be used to identify patients with low-risk gastrointestinal bleeds suitable for discharge and outpatient endoscopy. However, some debate remains regarding what level of Blatchford score can be considered low-risk. The aim of this study was to assess the need for intervention in patients presenting with upper gastrointestinal bleeding based upon the admission Blatchford score.

Methods All patients admitted with acute upper gastrointestinal bleeding to Sandwell and West Birmingham Hospitals NHS Trust from 1 January 2009 to 31 December 2009 were included in the study.

Results Overall, 470 patients with acute upper gastrointestinal bleeding were admitted during the study period. Of these $67.2 \%$ were male and $32.8 \%$ female. The mean age of patients was $64.0 \pm 18.8$ years. The most common diagnosis was peptic ulcer disease, which was found in $34.5 \%$ of patients. A Blatchford score of 0 accounted for $6.0 \%$ of patients $(n=28)$ and $14.7 \%(n=69)$ had a Blatchford score $\leq 2$. Of the patients admitted with a Blatchford score $\leq 2$ none required intervention (transfusion, endoscopic therapy or surgery) and there were no deaths. These patients were significantly younger than patients with a Blatchford score $>2$ (mean age $44.1 \pm 17.5$ years for a Blatchford score $\leq 2$ vs $67.4 \pm 18.8$ years for a Blatchford score $>2$ ).

Conclusion Patients with acute upper gastrointestinal bleeding with a Blatchford score $\leq 2$ did not require inpatient intervention and can be considered for early discharge from hospital with outpatient endoscopy. This strategy identified $14.7 \%$ of patients in our population that were unnecessarily admitted. Using a Blatchford score of $\leq 2$ may help to significantly reduce hospital admissions.

Competing interests None Declared.

\section{PM0-203 DIRECT GASTRIC PUNCTURE AND GASTROPEXY (FREKA ${ }^{\circledR}$ PEXACT) INSERTION TECHNIQUE FOR PERCUTANEOUS ENDOSCOPIC GASTROSTOMY REDUCES PERISTOMAL INFECTION RATES COMPARED WITH STANDARD PULL- THROUGH INSERTION IN PATIENTS WITH HEAD AND NECK CANCER}

doi:10.1136/gutjnl-2012-302514b.203

B R Disney, ${ }^{*}$ M Nizamuddin, A Tanajura, M Anderson, M Lewis. Gastroenterology, Sandwell and West Birmingham Hospitals NHS Trust, Birmingham, UK

Introduction Patients with head and neck cancer often have a percutaneous endoscopic gastrostomy (PEG) inserted to provide nutritional support while undergoing treatment. The standard pullthrough PEG technique is associated with a high incidence of peristomal infection. This is thought to be a result of pulling the PEG through the oral cavity which may be colonised with bacteria. In addition there is the risk of tumour seeding at the PEG site with this method. In our institution, such patients now have an endoscopical controlled introducer PEG (Freka ${ }^{\circledR}$ Pexact) with dual gastropexy inserted which avoids passage of the bumper through the oral cavity. We aimed to compare peristomal infection rates between the two methods of PEG insertion.

Methods We carried out a retrospective audit of PEG insertions in patients with head and neck cancer. Patients were identified using the $\mathrm{ADAM}^{\circledR}$ medical documentation system (Fujinon Europe $\mathrm{GmbH}$, Willich, Germany) and the Nutrition team logs. Complications, peristomal infection and 30-day mortality were documented after review of case notes and liaison with Community Nutrition Nurses.

Results A standard pull-through PEG (16F Corflo ${ }^{\circledR}$, Merck, UK) was inserted in 13 patients and 30 patients had a Freka ${ }^{\circledR}$ Pexact 15F (Fresenius Kabi, Germany) inserted. Of the Pexact group $76.7 \%$ were male $(n=23) ; 84.6 \%(n=11)$ of the standard group were male. The mean age of patients was 58 years (range $35-81$ ) in the Pexact group and 61 years (range $35-78$ ) in the standard PEG group. Prophylactic antibiotics were prescribed to $83.3 \%(n=25)$ in the Pexact group compared with $100 \%(n=13)$ of the standard pull-through PEG group. In the standard PEG group 69.2\% (n=9) developed peristomal infection compared with $36.7 \%(n=11)$ in the Pexact group. Immediate complications occurred in $15.4 \%(n=2)$ in the standard group and in none of those in the Pexact group. There were no deaths in either group at 30 days.

Conclusion The introduction of the direct gastric puncture and gastropexy technique led to a significant reduction of peristomal infections in patient with head and neck cancer. This new technique is well tolerated by patients.

Competing interests None declared.

\section{PMO-204 ASSESSING RISK OF ADVERSE OUTCOME IN ACUTE LOWER GASTROINTESTINAL BLEEDING: ARTIFICIAL NEURAL NETWORK VS SIGN GUIDELINES AND BLEED SCORE}

doi:10.1136/gutjnl-2012-302514b.204

${ }^{1} \mathrm{C}$ H Choi, ${ }^{2} \mathrm{~J}$ Swingland, ${ }^{1} \mathrm{~A}$ Ali, ${ }^{2} \mathrm{~S}$ Bose, ${ }^{1} \mathrm{~L}$ Ayaru. ${ }^{1}$ Department of Gastroenterology, Charing Cross and Hammersmith Hospitals, Imperial College Healthcare NHS Trust, London, UK; ${ }^{2}$ PET Methodology Group, MRC Clinical Science Centre, Imperial College London, London, UK

Introduction The majority of patients with acute lower gastrointestinal bleeding (ALGIB) have a low risk of requiring intervention, rebleeding or death. Nevertheless in routine clinical practice most are admitted to hospital for observation and endoscopy increasing cost of care. There are no risk scores routinely used in clinical practice which differentiate high risk patients who should be admitted to hospital from those who could be managed as outpatients. British Society of Gastroenterology/Scottish intercollegiate guidelines network (SIGN) have published expert opinion based criteria for non-admission but the accuracy of these is unclear.

Methods The aim of this study was to compare an artificial neural network's (ANN) performance in distinguishing high-risk from lowrisk patients with ALGIB to SIGN guidelines (six clinical variables) and BLEED score (five clinical variables). Data were collected retrospectively from patients with ALGIB who were admitted to the emergency department of a teaching hospital between 2007 and $2010(n=174)$. A multi-layered perceptron ANN model using back propagation and logistic activation function with hidden nodes to 